\title{
Ngoutidé, « l'illuminé de Lioua ». Destruction de fétiches et représentations de la sorcellerie en Centrafrique
}

Ngoutide" "the Enlightened One of Lioua". Destruction of Fetishes and Representations of Witchcraft in the Central African Republic

\section{Andrea Ceriana Mayneri}

\section{OpenEdition \\ Journals}

Édition électronique

URL : http://journals.openedition.org/etudesafricaines/17823

DOI : 10.4000/etudesafricaines. 17823

ISSN : $1777-5353$

Éditeur

Éditions de l'EHESS

Édition imprimée

Date de publication : 3 octobre 2014

Pagination : 739-768

ISSN : 0008-0055

Référence électronique

Andrea Ceriana Mayneri, « Ngoutidé, « l'illuminé de Lioua ». Destruction de fétiches et représentations de la sorcellerie en Centrafrique », Cahiers d'études africaines [En ligne], 215 | 2014, mis en ligne le 02 octobre 2016, consulté le 16 décembre 2020. URL : http://journals.openedition.org/etudesafricaines/ 17823 ; DOI : https://doi.org/10.4000/etudesafricaines.17823 


\section{Ngoutidé, «l'illuminé de Lioua » Destruction de fétiches et représentations de la sorcellerie en Centrafrique*}

Au cœur de la Centrafrique, dans une région peuplée massivement par différents groupes banda, se développe une formidable effervescence antifétichiste entre 1962 et 1966. Un certain Raymond Gonemba-Obal, mieux connu sous le nom de Ngoutidé, s'adonne à une œuvre de destruction de fétiches, parcourant des centaines de kilomètres et attirant des milliers de personnes vers le village où il réside. Il lutte contre les membres des associations initiatiques banda et prêche la conversion au catholicisme. Cette campagne de destruction de fétiches demeure, à notre connaissance, inconnue de la littérature anthropologique et historique portant sur la Centrafrique.

En nous appuyant sur nos recherches ethnographiques et sur des documents inédits des Archives des pères Spiritains à Chevilly-Larue ${ }^{1}$, nous tenterons de reconstituer la trajectoire biographique de cette figure hors-norme, en restituant le sens de ces événements dans le contexte des années 1960, et dans leur rapport à d'autres trajectoires anti-fétichistes qui ont vu le jour dans la région équatoriale. En reconstituant le parcours de Ngoutidé nous écouterons diverses voix : elles correspondent aux récits et aux souvenirs des personnes qui ont connu le prophète banda, de ses parents et des hommes religieux qui ont croisé son chemin. L'une de ces voix nous échappe cependant : celle de Ngoutidé lui-même, que nous n'entendrons jamais. La voix de celui que les Banda considèrent comme un " prophète $»^{2} s$ 'est éteinte avec

* Nous tenons à remercier Jan-Lodewijk Grootaers pour ses observations sur une première version de ce texte, ainsi que Sandra Fancello et Ramon Sarró pour leurs commentaires critiques sur notre interprétation de la figure de Ngoutidé. Nous remercions également le père Louis Sénéchal (Congrégation du Saint-Esprit) qui nous a communiqué ses souvenirs de Ngoutidé, et Wiel Eggen (Société des missions africaines) qui a repris avec nous certaines de ses notes de terrain des années 1970.

1. Archives générales de la Congrégation du Saint-Esprit (AGCdSE).

2. Dans le pays banda, Ngoutidé est désigné communément comme «le prophète banda international » et «le plus grand des Banda». Nous verrons que la trajectoire biographique de Ngoutidé est une conversion career qui n'a jamais donné lieu à un vrai prophétisme : le «ngoutidisme» n'a jamais existé. En utilisant le mot « prophète » nous renvoyons exclusivement au terme employé par les Banda, dans l'interprétation qu'ils proposent aujourd'hui de ces événements. 
sa mort, presque douze ans avant le début de nos recherches en Centrafrique ${ }^{3}$. Elle se tait surtout un jour inconnu à la fin des années 1960 lorsque Ngoutidé arrêta soudainement sa prédication parmi les Banda et que les récits de sa vie commencèrent à se répandre. Cette voix qui nous échappe n'est pas seulement orale. Son silence renvoie au manque d'informations sur le sens de la mission dont Ngoutidé se disait investi, ainsi que sur les temps et les moyens de son accomplissement en l'espace de quelques années. La reconstitution historique du parcours de conversion et de prédication de Ngoutidé se heurte à la «consommation» de son image par la population, qui en a fait l'objet d'une mythification aussi bien que d'une mystification. Ce qui peut ressortir d'authentiquement biographique dans ces pages permettra tout de même d'éclairer une figure et un événement historiques majeurs de cette région centrafricaine. Cependant, nos objectifs ne sont que partiellement biographiques. Prenant appui sur la distinction proposée par Douglas H. Johnson (1994 : ix, 351-352) selon laquelle une étude des prophétismes doit aborder, au-delà du versant biographique, l'historiographie du message prophétique, c'est-à-dire sa trajectoire historique et la façon dont son souvenir et ses interprétations changent dans le temps et ne cessent d'être reformulés, nous tenterons de discerner, entre les lignes du récit de la vie de Ngoutidé, les discours ordinaires à travers lesquels les Banda expriment leurs inquiétudes et leurs aspirations actuelles. En particulier, dans ces discours, la dénonciation de la recrudescence des malheurs d'origine sorcellaire sera analysée à la lumière du sentiment, largement répandu parmi les Banda, d'avoir été « dépossédés » des connaissances et pouvoirs des ancêtres. Nous interrogerons donc la manière dont la mémoire de Ngoutidé et la transmission du récit de son œuvre de destruction de fétiches participent d'une « réinvention » du passé récent à travers laquelle les Banda continuent de réfléchir sur les dynamiques historiques qui les ont amenés, au long du $\mathrm{XX}^{\mathrm{e}}$ siècle, à abandonner les cultes traditionnels et à adhérer à la religion des missionnaires.

\section{Les Banda de Centrafrique}

La crise centrafricaine - dont le coup d'État du 24 mars 2013 à travers lequel la coalition Séléka a renversé le pouvoir du général Bozizé n’est que le dernier épisode en date — s'inscrit dans une histoire longue de violence

3. Les données ethnographiques ont été recueillies au cours de plusieurs séjours de terrain en Centrafrique entre 2005 et 2012 et complétées par des recherches d'archives à Bangui et en France. En Centrafrique, nous travaillons dans une vaste région peuplée majoritairement par les Banda. Du point de vue administratif, cette région correspond largement à la Préfecture de la Ouaka, au centre du pays, dont la ville de Bambari est le chef-lieu. Pour l'essentiel, la chronologie de la vie de Ngoutidé demeure imprécise, y compris en ce qui concerne la date exacte de son décès. Nous y reviendrons. 
et de coercition dont les origines remontent, en partie, aux dynamiques mises en branle par la colonisation et, bien avant, aux déplacements de populations imposés par la traite esclavagiste orientale (Marchal 2009). Les Banda, aujourd'hui établis dans une vaste région au cœur même de la Centrafrique, sont originaires des régions plus septentrionales, où ils connurent une migration massive tout au long du XIX ${ }^{\mathrm{e}}$ siècle, en descendant vers la partie centrale du seuil Oubangui-Chari (Santandrea 1964 : 315 ; Eggen 1976 : 11/a, 12/a-b ; Cordell 1985 : 26). Comme d'autres populations, ils tentaient ainsi d'échapper aux razzias des négriers en provenance du Wadai et du Bagirmi, puis des marchands d'ivoire et d'esclaves en provenance du Soudan (Cordell 1985 : 28). Ayant été confrontés jusqu'au début du $\mathrm{XX}^{\mathrm{e}}$ siècle aux raids menés par le sultan du Dar al-Kuti Muhammad al-Sanusi, les groupes banda se heurtèrent aussi aux populations déjà établies sur le territoire, dont les royaumes zandé et nzakara du bord de la rivière Oubangui, et aux groupes manza et gbaya de l'Ouest. À l'arrivée des premières missions d'explorations européennes, les Banda étaient donc depuis longtemps des « réfugiés avant l'heure » (ibid. 1999: 17) et, comme d'autres populations équatoriales, «disséminés en villages dépourvus d'une organisation centrale susceptible de résister aux exigences sans cesse accrues des missions de pénétration » (Coquery-Vidrovitch 1972 : 75). Le régime concessionnaire d'abord, et l'administration coloniale ensuite, agirent de façon décisive sur l'affaiblissement de l'organisation socio-politique banda, que Wiel Eggen (1976: 45/d) ${ }^{4}$ a décrit comme un «système politique à assise religieuse » où l'autorité et le pouvoir reposaient sur l'appartenance à un groupe de parenté et sur la possession de dons d'ordre moral et religieux. Parmi ces «dons », un prestige particulier découlait de l'affiliation aux associations initiatiques, dont la protection était recherchée et l'autorité était respectée autant qu'elle était redoutée de la part des non-initiés (ibid. : 11/f-h). Parallèlement à l'implantation de l'administration coloniale, les premières missions religieuses amorcèrent leur pénétration du pays : parmi les Banda, la mission catholique Saint Joseph de Bambari fut construite entre 1914 et 1920 (Toso 1994 : 1520). Mais le père spiritain Joseph Daigre sillonnait le pays banda déjà en 1907, suivi, quelques années plus tard, par son confrère Charles Tisserant : tous les deux nous ont laissé les premières observations ethnographiques sur la culture et la langue banda.

Le 27 mars 1938, Barthélémy Boganda, le premier prêtre oubanguien, était ordonné à Bangui. Il devait devenir par la suite une figure de premier plan dans la vie politique du pays et, en 1958, le président du gouvernement centrafricain (Kalck 1995). Affecté en 1941 à la mission de Bambari, l'abbé

4. À la demande de la Conférence épiscopale centrafricaine, Wiel Eggen effectua dans les années 1970 des enquêtes ethnographiques parmi les Banda, dans une région qui correspond largement à notre terrain de recherche. En 1976, sous les auspices de Pro Mundi Vita, il écrivit Peuple d'autrui, une thèse non publiée sur la culture et l'histoire banda. 
Boganda fut nommé responsable du secteur compris entre les localités de Grimari, Bakala et Kouango, qui s'étendent en éventail du nord au sud de Bambari (Pénel 1995: 23). La correspondance de Boganda, écrite de Bambari et conservée actuellement aux Archives spiritaines, démontre que l'abbé menait des tournées anti-fétichistes dans les villages aux alentours de Bambari, et que son action visait en particulier à dissoudre l'association initiatique semalì, dont les affiliés vouaient un culte à l'esprit de la brousse Ngakola. Soumis à la pression de certains de ses supérieurs (ibid: 39), et de plus en plus en conflit avec les missionnaires spiritains à cause de leur proximité avec l'administration coloniale (Kinata 2008 : 556-562), l'abbé s'engagea dans les années suivantes dans une carrière politique fulgurante. En 1958, il approuvait le projet du général de Gaulle sur la communauté et proclamait à Bangui une République centrafricaine en prenant la tête du gouvernement (Kalck 1992 : 300). Mais Boganda ne put assister à l'aboutissement de ses efforts : le 29 mars 1959, l'avion dans lequel il voyageait s'écrasait au sol en rentrant à Bangui. Le 13 août 1960, le pays accédait à l'indépendance en pleurant la disparition de son principal inspirateur.

C'est peu après l'accès à l'indépendance, dans le vide laissé par la disparition de Boganda que, dans un village de la Ouaka - où l'abbé, vingt ans plus tôt, avait prêché et lutté contre le « fétichisme » — un certain Raymond Gonemba-Obal se réveilla à la suite d'une longue maladie et qu'il entama une mission anti-fétichiste.

\section{Un prophète en pays banda}

Raymond Gonemba-Obal naquit vers 1939 dans le village de Kossamba, entre les villes de Bambari et Kouango ${ }^{5}$. Lorsqu'il était encore très jeune, son père mourut et Raymond grandit auprès d'un oncle à Bambari. Là, il demeura dans un quartier proche de la Cathédrale St. Joseph. Plus tard, il fut employé comme manœuvre dans les transports publics. Il se maria une première fois, et le jeune couple essaya en vain d'avoir un enfant. C'est probablement à cette époque que Raymond manifesta les premiers symptômes d'une maladie qui aujourd'hui, dans la Ouaka, est communément décrite en français comme une "paralysie » ou un «état cataleptique ». Raymond fut affecté par les transports publics à Alindao, à 120 kilomètres au sud-est de Bambari. Sa femme dut l'hospitaliser une première fois. Il fut par la suite ramené à l'hôpital de Bambari et, enfin, à Lioua, un petit village à une dizaine de kilomètres de Bambari où il habita la maison d'un

5. Cette reconstitution du parcours de Ngoutidé est basée sur les nombreux récits recueillis entre 2005 et 2012 dans la Ouaka et à Bangui, les données tirées d'un cahier manuscrit conservé au village Lioua et les écrits des missionnaires conservés dans les Archives spiritaines. Le cahier de Lioua contient une dizaine de pages intitulées «Histoire de Gonemba-Raymond», écrites en sango le 3 décembre 1994 par un catéchiste du village. 
frère de sa femme. Raymond était alors immobilisé : il ne parlait plus et ne bougeait pas de son lit. Il n'était plus capable de s'alimenter ni de se laver. Sa femme Marie s'occupait de lui. Des récits oraux que nous avons recueillis dans la Ouaka font référence à un trouble mental. Il est précisé qu'il aurait refusé à plusieurs reprises des soins traditionnels, ce qui exclut l'inconscience. Il est cependant impossible d'élucider le caractère propre de cette maladie. L'idée d'un trouble mental correspond à une seconde maladie qui accompagnera Raymond dans les derniers mois de sa vie, et sur laquelle nous reviendrons ${ }^{6}$. Le refus des soins traditionnels est certainement un détail signifiant du récit qui renvoie au réveil anti-fétichiste qui attendait Raymond. À Lioua, on décida de le ramener à Kossamba, son village natal. La mère de Raymond fit appel à un certain « père Henry » de la mission de Kouango, de passage dans la région de $\mathrm{Kossamba}^{7}$. Ce dernier rendit visite au malade et lui donna le baptême en guise d'extrême-onction. Nous sommes probablement vers la fin de l'année $1962^{8}$.

Nous lisons sur les pages écrites en 1964 par un missionnaire catholique affecté à Bakala que lorsque le «père Henry » rejoignit le village de Raymond, « il s'arrêta et constata que ce malade était à la dernière extrémité. Il l'instruisit rapidement des vérités les plus importantes de la religion et lui donna le baptême. À peine cet homme eut-il reçu ce sacrement qu'il commença à aller mieux. Il ne mourut pas comme prévu et fut rapidement guéri ${ }^{9}$. En effet, peu après avoir reçu le baptême, Raymond bougea un pied, puis les mains et enfin il se dressa sur son lit. Quelques jours plus tard, il recommença à parler et il se rendit à l'église du village. Il s'agenouilla et il resta longtemps dans cette position. Ce n'est que le soir venu qu'il se leva. Il était suivi par tous les gens de son village.

Comme pour la maladie, il est difficile de clarifier les différentes phases de la guérison. Des témoignages recueillis au village Lioua insistent sur la faiblesse d'un corps épuisé par l'immobilité et sur la souffrance de Raymond dans sa marche vers l'église, d'autres introduisent une nouvelle figure, une femme appelée Ndembele qui aurait prié à côté de Raymond après le passage du père Henry ${ }^{10}$. Ce n'est qu'après cette deuxième prière, selon ces témoignages, que Raymond recommença à parler. Enfin, lorsque Raymond

6. Selon le père Louis Sénéchal, qui connut le prophète et officia lors de ses funérailles, «sa première maladie était bizarre tout comme la seconde : c'était un trouble psychique » (communication personnelle, 25 juin 2007).

7. Il s'agit du père Henri van den Berg de la Congrégation du Saint-Esprit, en Centrafrique de 1949 à 1979 (communication personnelle du père Louis Sénéchal, 25 juin 2007). Sa présence est effectivement signalée à Kouango par Toso (1994 : 247-248).

8. Selon le troisième frère cadet de Raymond, que nous avons rencontré à Bambari, et qui nous a raconté la vie de son frère en se référant à des notes prises sur un cahier, cette maladie aurait duré trois ans.

9. Journal de Bakala du 31 décembre 1964, AGCdSE, chemise 5J2.1.

10. La présence de Ndembele est citée dans le cahier intitulé « Histoire de GonembaRaymond » et confirmée par Mathias, le frère de Raymond. 
rejoignit l'église, il aurait prononcé pour la première fois des mots dans une langue mystérieuse, que son frère cadet définit lors de notre entretien « comme de l'hébreu »"${ }^{11}$.

Selon les témoignages recueillis à Lioua auprès de membres de sa famille, dans les jours qui suivirent Raymond se rendit dans «la maison de son père » — probablement chez un oncle paternel — où il ramassa des « fétiches ». Puis, il prit les «fétiches que les gens cachent dans la brousse », il sortit de la brousse avec «les fétiches que les gens usent pour tuer»et, enfin, « les gens qui ont caché des fétiches [...] vont vers lui avec ça ». Raymond avait commencé sa mission. Il adopta alors, ou plutôt les gens de Kossamba lui attribuèrent, le nom de «Ngoutidé » qui, en sango, la langue véhiculaire de Centrafrique, signifie « eau froide».

Ngoutidé choisit la croix comme symbole de sa prédication. Il quitta Kossamba et, dans les villages où il arrivait, il faisait ériger des croix. Parfois, il remplaçait les anciens fétiches avec de petites croix en bois qu'il donnait aux personnes qui l'approchaient. Il n'acceptait pas d'argent, sauf pour acheter les aliments qui étaient l'objet d'interdits alimentaires et que, ensuite, il proposait de préparer et de manger sans crainte. Les témoignages recueillis insistent sur l'opposition que Ngoutidé aurait rencontrée de la part des autorités administratives et de certains membres de l'Église catholique. Ainsi, il aurait été emprisonné, on lui aurait enlevé sa croix « pour l'envoyer à Rome » et il aurait été hospitalisé pour être «analysé ». Dans les pages des journaux de mission déjà cités, les catholiques qui assistent au passage de Ngoutidé ne manifestent pas d'hostilité à l'égard de sa prédication, au contraire, le missionnaire affecté à Bakala écrivait explicitement qu'il « approuvait» l'action de Ngoutidé ${ }^{12}$.

Le Journal de Bakala du mois d'octobre 1965 contient cette annotation : «Depuis quelques jours Ngoutidé est à Bakala. Il est venu assister à la grande messe aujourd'hui et sa présence a amené une centaine de personnes en plus à la messe ${ }^{13}$. Et cinq mois auparavant, dans le Journal de la communauté de Grimari, ville située à 80 kilomètres de Bakala, une autre annotation signalait non sans excitation : "Grimari connaît une grande animation; de toutes les routes des "pèlerins" viennent se faire "bénir" par le fameux "Ngoutidé". Païens — et chrétiens ! — vont faire enlever leurs tabous, leurs yoro! Aubale — c'est le vrai nom de Ngoutidé — invite ses clients à suivre la Mission catholique. Son emblème est la Croix que l'on

11. Il s'agit ici d'une opinion personnelle du frère de Raymond, dont il faudra surtout retenir la référence à une langue qui est associée à maintes figures prophétiques de l'Ancien Testament. En effet, les quelques mots de cette langue mystérieuse que le frère de Raymond se rappelle encore n'entretiennent aucun rapport, à notre connaissance, avec l'hébreu (c'est le cas, par exemple, du mot sordang pour désigner le pain).

12. Journal de Bakala du 31 décembre 1964.

13. Journal de Bakala du 10 octobre 1965. 
peut voir partout, dans les cases, devant les habitations ${ }^{14}$. Le missionnaire affecté à Grimari attribue une connotation religieuse marquée à la visite de Ngoutidé dans la localité : il parle de pèlerinage et de bénédiction, mais il mentionne aussi les yoro que la population remettait au prophète. Le mot sango yoro revêt plusieurs significations : il peut être à la fois un médicament, un remède, un poison, et plus généralement «tout objet naturel ou fabriqué, doté de pouvoirs curatifs, magiques, apotropaïques ou destructeurs » (Bouquiaux 1978 : 386). Son équivalent en langue banda est l'ayo, dont la polysémie était signalée par le père Tisserant (1931 : 364) lorsqu'il écrivait, dans son Dictionnaire Banda-Français publié en 1931, qu'il peut être "ce que l'on tire de l'arbre », donc un poison ou un remède d'origine végétale, mais aussi «la représentation matérielle des esprits [...] statues plus ou moins complètes [...] amulette-remède », et finalement la «pratique fétichiste servant de remède ». Le yoro et l'ayo désignent donc un ensemble de substances, de remèdes, de pratiques et d'objets sacrés auxquels il est impossible d'attribuer une connotation exclusivement positive ou négative, sans considérer l'intention et la finalité de leur utilisation : aujourd'hui, la traduction de ces termes par le mot français « fétiche », repris par la population, véhicule toutes les contradictions de «l'étonnante histoire théorique » du concept de fétiche (Pietz 2005 : 7).

Comme nous l'avons vu, d'après les récits diffusés à Bambari et dans la préfecture de la Ouaka, Ngoutidé pénétrait dans la brousse et il en sortait avec des fétiches, ou des gens allaient vers lui en lui remettant leurs fétiches « cachés dans la brousse » pour qu'il les brûle. Ces détails se réfèrent certainement au bada, le camp situé à l'écart du village dans lequel se réunissaient les adeptes du culte semalì voué à Ngakola. Dans ce camp bada, des représentations matérielles de l'esprit Ngakola, de sa femme Yamissi et de leur fille Ngandala étaient cachées. C'est dans le bada que les initiations au culte semali, s'étalant parfois sur plusieurs mois, avaient lieu. Et c'est précisément contre le culte semalì de Ngakola que l'abbé Boganda avait écrit en 1943, en le classant parmi les «monstruosités » qui dégradaient le peuple banda: «Ici rien d'extraordinaire, toujours les mêmes difficultés [...]. Il faudra refuser aux sacrements un chrétien qui [...] vient de prendre une deuxième femme ou de renvoyer sa femme légitime ou de se faire initier au "somali" [...]. La plus grosse besogne chez le Banda consiste à réagir [...] contre le divorce, la polygamie et le fétichisme sous ses mille formes ${ }^{15}$. Et vingt ans plus tard, le missionnaire affecté à Bakala écrivait dans son Journal : «[Ngoutidé] se donna pour mission de supprimer les fétiches et tout ce qui sert au culte des esprits et du démon $»^{16}$.

14. Journal de communauté de Grimari, AGCdSE, chemise 5J2.6a, mai 1965.

15. Barthélémy Boganda, lettre manuscrite, Bambari, le 21 décembre 1943. AGCdSE, Fond de Banville, chemise 46.11.

16. Journal de Bakala du 31 décembre 1964. 




En 1964, ce que le missionnaire considérait comme un «mouvement de conversion » était donc à son apogée. Dans le Journal de la mission nous pouvons lire :

«Il a suffi que Ngoutidé ouvre la bouche pour qu'on lui obéisse. Il n'a appelé personne et les routes sont pleines de gens allant spontanément vers lui, emportant dans leur baluchon leurs yoro transmis par leurs ancêtres et destinés maintenant à être jetés au feu. Beaucoup font à pied cent et même deux cents kilomètres pour accomplir leur pèlerinage. Il y en a qui sont morts de fatigue sur la route. L'hôpital de Bambari recueille ceux qui se sont blessé les pieds sur les pierres de la route et ne peuvent plus marcher. »

En 1965, le mouvement atteint Grimari, en suivant les déplacements de Ngoutidé. Pour rejoindre Bakala et Grimari, Ngoutidé est nécessairement passé par Bambari. Il est probable que sa renommée se soit étendue dans les régions du Sud-Est, peuplées par les Banda yakpwa. Un témoignage recueilli en 2007 à Bangui parle de «pèlerins » en provenance de Bamingui, à quelque 300 kilomètres au nord de Bambari ${ }^{17}$ : le nom de Ngoutidé semble avoir circulé dans une vaste région. Entre 1965 et 1966, en revanche, le mouvement semble s'affaiblir, du moins dans la région comprise entre Bambari, Bakala et Grimari. Le Journal de Bakala du 25 décembre 1965 raconte :

17. Entretien avec le Dr Zoumbala, originaire de Bamingui (Bangui, 4 août 2007). 


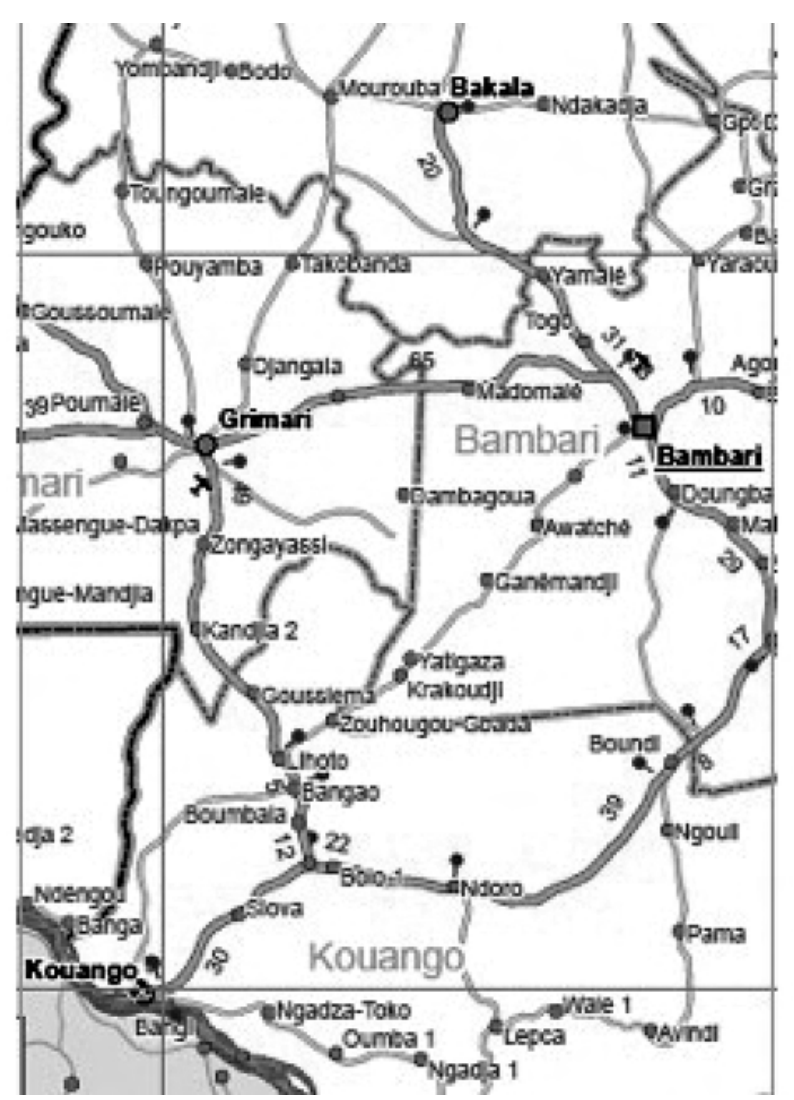

La Centrafrique (Section de la cartographie des Nations Unies, carte n. 4048, rev. 6, avril 2013) et la Préfecture de la Ouaka (OCHA Centrafrique) : la distance qui sépare Bambari de Bakala est de $80 \mathrm{~km}$. Le village Lioua se trouve à $10 \mathrm{~km}$ à l'est de Bambari. De l'autre côté de l'Oubangui, la République démocratique du Congo.

« Pendant la nuit de Noël un groupe de protestants a démoli les croix élevées dans le village de la rive droite. Les gens furieux parlent de mettre le feu à la Mission protestante ${ }^{18}$. J'essaie de les calmer. En allant voir un malade sur la rive droite je constate que les protestants n'ont pas touché aux croix élevées à l'emplacement du catéchisme. Ils ont brisé les croix plantées par Ngoutidé. »

$\mathrm{Au}$ mois de septembre 1966, à Bakala, il ne reste plus que l'écho du passage de Ngoutidé. Les croix qu'il avait érigées ont été détruites par les membres de la Mission protestante. Au moment où il arrêta sa mission, Ngoutidé s'installa au village Lioua, où il avait été amené lors de sa première maladie. Là il bâtit une grande maison « en dur ». Selon le père Louis

18. Il s'agit ici de la Baptist Mid-Mission dont l'implantation dans la Ouaka succéda à celle de la mission catholique. Voir la note 29. 
Sénéchal, il ne parlait «absolument jamais de son travail prophétique ${ }^{19}$. La légende qui l'entoure relate qu'il obtint des succès remarquables dans les compétitions de vélo. Effectivement, en 2007, dans l'ancienne maison de Raymond nous avons repéré une attestation faite à Bangui le 19 juin 1972 :

« Le Président de la République - Grand Maître de l'Ordre - sur proposition du Ministre de l'Éducation Nationale, de la Jeunesse, des Sports, des Arts et de la Culture - a décerné à Monsieur Gonemba-Obal Raymond, Capitaine du Club Vélocipédique à Bambari, la Médaille de bronze du Mérite Sportif. »

Au mois d'octobre 1991 se déclencha la seconde « crise » de Raymond. Elle durera deux ans, jusqu'à sa mort. Sur la route qui conduit à Lioua, Raymond rencontra des femmes de la congrégation catholique des Légionnaires de Marie. Il marcha à côté d'elles. Après une vingtaine de kilomètres, avant d'arriver au village, Raymond fut de nouveau «paralysé ». Comme dans les années 1960, les efforts des médecins de Bambari ne donnèrent aucun résultat ${ }^{20}$. Raymond demeura immobilisé dans son lit, mais il parlait encore et il demanda au père Louis Sénéchal le baptême ainsi qu'un mariage catholique avec sa dernière femme. Le 25 février 1992, Raymond se leva une dernière fois : il tenta de frapper sa femme et marcha à demi-nu de long en large dans la cour de la maison. Puis il se rendit chez son frère cadet et il détruisit son poste de radio et sa machine à coudre. Il brandit une lance. Lorsque sa femme réussit à le calmer, Raymond se laissa ramener au lit. Il mourut peu de temps après ${ }^{21}$.

\section{L'événement Ngoutidé22}

L'itinéraire biographique de Raymond «Ngoutidé »s'inscrit dans le sillage d'autres mouvements anti-fétichistes qui ont surgi et qui continuent de surgir en Afrique. Le récit de cet itinéraire est d'ailleurs structuré autour d'un canevas fixe, énoncé de manière presque invariable, insistant sur les événements qui transfigurent une existence, certainement particulière, en destin :

19. Communication personnelle (25 juin 2007).

20. Au mois de décembre 2008 nous avons contacté le docteur Fabrice Simon, à Marseille. À l'époque du décès de Ngoutidé le docteur Simon était affecté à l'hôpital universitaire de Bambari et certains témoins dans la Ouaka le citent comme le médecin consulté lors de la dernière maladie du prophète. Bien que «le nom ne lui est pas inconnu », il ne se souvient de rien à propos de ce patient en particulier (communications personnelles, 28 et 29 décembre 2008).

21. Les événements des années 1990 sont reconstruits à partir des souvenirs personnels du père Louis Sénéchal qui assista aussi à ce dernier épisode de «folie » de Raymond. Il est cependant impossible de préciser la date de sa mort : aucun document dans les archives de Bambari ne l'évoque, tandis que sur la tombe de Raymond, une date écrite en blanc est désormais illisible.

22. L'expression l' «événement Ngoutidé » est empruntée à Jean-Pierre DozoN (1995: 74) qui définit le prophète Harris comme un «personnage-événement ». 
la maladie et la guérison, la lutte contre les fétiches et les compétitions en vélo, la dernière maladie et la mort du prophète. La matrice de cette expérience prophétique centrafricaine est commune à ceux qu'André Mary (2010 : 231) appelle les «proto-prophètes » africains, tels que W. W. Harris et Simon Kimbangu, et que l'on retrouve dans les itinéraires de vocation des petits prophètes d'Eboga des années 1950 au Gabon (ibid. : 236-237), notamment par certains détails qui précèdent la crise, l'état cataleptique, la maladie ou la conversion de ces figures hors-norme. Nous nous référons ici, et malgré des différences évidentes entre ces expériences distantes dans le temps et l'espace, aux rapports ambivalents avec le milieu des missions chrétiennes, auxquels s'ajoute l'expérience d'un voyage ou déplacement qui contribue à distancier ces jeunes de l'espace social villageois et à entrer en contact avec le monde urbain ou le travail salarié. Nous savons par exemple que Harris, né au Liberia vers 1860 d'une mère chrétienne dans une famille traditionnelle et «païenne » (Shank 1994: 41), et qui faisait lui-même remonter sa «conversion» à ses 21 ans, quitta son village natal une première fois à douze ans en suivant son oncle, le pasteur méthodiste John C. Lowrie à côté duquel il s'immergea dans un environnement plutôt occidentalisé en apprenant l'anglais et en étudiant la Bible (ibid. : 47-49), puis une deuxième fois vers ses 19 ans en s'employant sur les bateaux qui parcouraient la côte africaine jusqu'au Gabon. Simon Kimbangu, né vers 1890 dans la partie nord-orientale de la région culturelle kongo et confié aux missionnaires de la British Missionary Society, quitta son pays natal en 1919 pour se rendre à pied à Léopoldville, en vue d'échapper aux apparitions et à la voix qui l'appelaient à une mission particulière (Vellut 2005 : xii) et de se soustraire au conflit ouvert avec les doyens de sa communauté, sans exclure la recherche d'un travail mieux rémunéré (Asch 1983 : 20). À Léopoldville, Kimbangu entra en contact avec «le milieu urbain, avec des anciens provenant de sa région, mais aussi avec une élite congolaise qui avait voyagé [...] qui était alphabétisée, qui comptait l'un ou l'autre entrepreneur dans ses rangs » (Vellut 2005 : xii).

Dans la région centrafricaine de la Ouaka, au début des années 1940, Raymond, orphelin de père, quitte lui aussi son village natal pour suivre un oncle à Bambari, le centre urbain le plus important de la région : puisque nous savons que quelques années plus tard il a été employé des transports publics et que successivement, après la fin de sa prédication, il a entretenu de bons rapports avec des commerçants étrangers (probablement des Portugais $)^{23}$, on peut présumer qu'à Bambari le jeune Raymond ait fréquenté l'école locale, à l'époque gérée par l'Église catholique et dont l'abbé Boganda venait d'être nommé responsable en 1942. Or, à la fin des années 1950 nous retrouvons Raymond, désormais âgé d'une vingtaine d'années, gravement malade et en proie aux tourments d'une paralysie/folie qui n'exclut

23. Souvenir personnel du père Louis Sénéchal. 
pas le refus catégorique des remèdes traditionnels. Entretemps, nous ne pouvons qu'imaginer l'éducation de ce jeune banda, dans un contexte sur lequel les renseignements ethno-historiques sont particulièrement pauvres, mais qui vivait certainement les contradictions de la lutte acharnée contre les cultes traditionnels menée, parmi tant d'autres, par l'abbé Boganda, et qui assistait à l'émergence d'une conscience politique anticoloniale et nationaliste commençant alors à se dessiner dans l'Oubangui-Chari en passant précisément par le pays banda ${ }^{24}$. À côté des indices qui témoignent de la problématique de l'acculturation à l'occidentale, sur fond de télescopage du message chrétien et des formes de pensée traditionnelles, on devine les inquiétudes d'un parcours tumultueux dont le récit à travers les décennies retient comme principaux «symptômes » la cohabitation difficile avec son épouse et la maladie. C'est d'ailleurs un destin commun à plusieurs de ces figures d'échouer dans leurs tentatives d'épanouissement: c'est l'échec aux examens pour devenir pasteur qui s'exprimera pour Kimbangu par le besoin d'agir de manière autonome en marge de l'Église officielle, voire en s'y opposant (Balandier 1955 : 428). L'expérience de l'échec, parfois revécu dans le contexte familial ou traditionnel et dans le monde urbain et professionnel, accompagne effectivement les trajectoires des prophètes ngunza qui suivirent et réinterprétèrent le parcours de Kimbangu (MacGaffey 1983 : 102). Le parcours de Raymond semble cumuler ces expériences diverses. Le périple à travers un monde semi-urbain en plein devenir et les désarrois personnels constituent une propédeutique à l'apparition des signes d'élection classiques - la maladie et la folie, le retrait du monde et la catalepsie qui caractérisent les itinéraires de vocation prophétique divers comme ceux des prophètes nuer d'un côté, et celui des visionnaires du Bwiti fang de l'autre ${ }^{25}$ (Mary 2000 : 82-83). Nous assistons, en d'autres termes, au déploiement d'un scénario historique et initiatique, largement répandu dans d'autres contextes africains: un scénario qui se compose de la maladie et de la guérison, à laquelle vont succéder le « saut dans la vocation » et la confrontation avec des situations d'épreuve (Dozon 1995 : 195, 198), sachant, comme le soulignait déjà Desroche (1963 : 82-83) à la suite de Bastide, que la figure du prophète «raté » est presque «une loi du genre » et que cet itinéraire

24. C'est à Bambari, au début des années 1940, que Barthélémy Boganda enseigne le catéchisme au futur docteur Abel Goumba qui jouera un rôle de premier plan dans le parcours vers l'indépendance centrafricaine, puis dans la vie politique du pays, à travers l'expérience de l'exil, jusqu'à son décès en 2009. Nous avons aussi signalé le désaccord entre Boganda, à l'époque de son séjour parmi les Banda, et les représentants de l'Église catholique de la Ouaka, autour de la proximité entre les missionnaires et l'administration coloniale. Dans la Ouaka, entre 2005 et 2008, nous avons pu recueillir les derniers souvenirs d'anciens élèves de l'abbé Boganda, dont certains le suivirent dans son aventure politique à Bangui et à l'étranger. Sur la formation de Boganda, voir PÉnel (1995) et KALCK (1995: 50-52).

25. Mais la maladie, la gale en l'occurrence, joua également un rôle important dans la jeunesse de Kimbangu (Vellut 2010 : 20). 
biographique tend inéluctablement à déboucher sur une situation d'impasse ou sur l'échec.

La conversion et la vocation qui attendent Raymond suivent un tracé qui est loin d'être aléatoire : si ces deux événements, ainsi que les modalités de la guérison et puis la prédication, se produisent dans le sillage de l'entreprise missionnaire catholique - de loin la plus présente dans le pays banda néanmoins c'est le surnom même de Raymond, « eau froide », qui renvoie à une expérience plus articulée. On ne peut que mentionner ici le récit que Harris lui-même fit de sa «pentecôte » au missionnaire Pierre Benoît, en 1926 : le prophète y retraçait les caractéristiques de la vision et visitation de l'ange Gabriel qu'il avait eues en prison en 1909, et décrivait l'onction de l'Esprit-Saint comme un jet d'eau, comme «de la glace sur ma tête, et sur toute ma peau » (Shank 1999 : 55). La référence à l'eau et à la fraîcheur est ainsi devenue un topos de l'expérience de la réception de l'Esprit-Saint dans la conversion pentecôtiste (Fancello 2006 : 105). La superposition de plusieurs registres confessionnels marque en effet la suite du parcours de Ngoutidé, sans qu'on puisse trancher la question de savoir si elle découle de l'initiative du prophète banda lui-même ou si elle s'est faite au fil des années à travers la réélaboration du récit. S'il est vrai que la mission dont Ngoutidé s'investit était solidaire de la longue tradition catholique dans la Ouaka, et qu'elle trouva une certaine opposition de la part des communautés protestantes dans la localité de Bakala, il faut cependant interroger, dans le discours prêté au jeune banda, les quelques références à la possession de dons ou de «charismes $»^{26}:$ la prédication d'abord, puis le début d'une pratique de guérison, et d'une forme de glossolalie qui, d'après le frère cadet et la dernière femme de Raymond, durera jusqu'à sa mort, mais aussi la «bénédiction » qu'il prononçait sur ceux qui l'approchaient (selon le missionnaire catholique de Grimari) et, finalement, la destruction des fétiches par le feu. Si l'implantation de l'Église catholique parmi les Banda demeure peu connue, la reconstitution de la pénétration protestante, et éventuellement la diffusion de mouvements messianiques, est plus ardue en raison de l'absence d'études et du silence des archives. Nous nous limiterons donc ici à signaler quelques faits qui mériteraient certainement de faire l'objet d'études ultérieures.

La présence protestante est attestée à la périphérie du pays banda par un souvenir de Michel Leiris (1934: 197) qui, voyageant avec la Mission Dakar-Djibouti, le 14 mars 1932, croise des «missionnaires américaines » se rendant de Fort-Sibut, en pays manza, à travers Bambari et quelques 150 kilomètres plus loin. À Bambari, en 1955, un missionnaire catholique signalait l'arrivée en ville des Témoins de Jéhovah, et un deuxième missionnaire signalait au début des années 1960 la présence des prophètes ngunzistes le long de la côte zaïroise de l'Oubangui sans fournir d'autres détails

26. Journal de Bakala du 31 décembre 1964. 
ou commentaires ${ }^{27}$. De nouveau du côté centrafricain de la rivière, non loin de la région où Raymond guérit et entama sa mission, Suzanne Jean (1963 : $50)^{28}$, diplômée de l'Institut d'ethnologie, envoyée en 1959 parmi les Banda langba par le Bureau pour le développement de la production agricole, observait comment, sous l'impulsion de celui qu'elle définissait comme un «évangéliste intelligent», la population abandonnait le culte semali de Ngakola, et comment les convertis au protestantisme se multipliaient. L'implantation d'une mission à Fort-Sibut, d'où les missionnaires américaines rencontrées par Leiris partaient pour des tournées en pays banda, remonte probablement à 1920 : il s'agit de la Baptist Mid-Mission, et il est certain que les protestants qui s'opposèrent au travail de Ngoutidé à Bakala en 1965 et 1966 appartenaient à cette dénomination ${ }^{29}$. En 1967, un séminaire Baptiste était ouvert à Bambari depuis quelques années. Les archives de la Faculté de théologie évangélique de Bangui, qui abritent pourtant des documents inédits d'un certain intérêt, ne nous offrent malheureusement pas de renseignements plus précis sur l'implantation et les activités de ces missions en pays banda. Le silence des sources catholiques est frappant et incite à penser qu'une distance se serait creusée dans le temps avec les missionnaires protestants et se serait accentuée après le mouvement déclenché par le passage de Ngoutidé. On peut supposer, en d'autres termes, qu'un antagonisme de longue date ait pu se consolider autour de l'interprétation de cette figure itinérante et de ses symboles, de manière similaire au processus que Jean-Luc Vellut (2010) a analysé dans le cas des dénominations baptistes américaines présentes au Congo au moment où Simon Kimbangu entamait son propre parcours prophétique. Sans oublier qu'une certaine proximité entre l'Église catholique et l'administration coloniale avait déjà été au cœur du contentieux qui avait opposé l'abbé Boganda à ses supérieurs, et que le sujet a constitué dans d'autres contextes coloniaux l'un des thèmes forts des dénonciations adressées par les missions protestantes à l'égard des relations entre les catholiques et le pouvoir institué (ibid. : 19-20). Il est probable que la prolifération des croix de Ngoutidé, celles plus petites qu'il distribuait lui-même et les plus grandes qui surgissaient devant les cases, ait été interprétée par les protestants comme une menace portée soudainement à l'intégrité de leur communauté, établie sur l'autre rive du cours d'eau qui traverse Bakala.

27. AGCdSE, Journal de la Communauté catholique de la Mission St. Joseph de Bambari, chemise 5J2.2a.

28. Ne pas confondre le village de Bambari mentionné par S. Jean dans son étude, proche de l'Oubangui dans ce qui était alors le district de Kembé, avec le cheflieu de la Ouaka auquel nous nous référons ici.

29. Nous remercions ici M. Juan Fandos-Rius pour nous avoir communiqué des informations tirées d'un texte en sa possession : Robert W. Hill, The Christianization of the Central African Republic, Fuller Theological Seminary, June 1969, pp. 116122. 
Dans la suite du parcours de Ngoutidé, nous assistons à la multiplication des signes qui contribuent à préciser les contours d'un personnage prophétique, qui agit selon le scénario classique (Mary 2000: 83), et qui le conduira d'un village à l'autre en brûlant les fétiches : «Un des premiers messages des récits de conversion, écrit A. Mary (2010 : 132), est d'attester la rupture avec les voies de l'initiation aux cultes traditionnels [...]. Se convertir c'est se repentir, rompre avec un passé inavouable et pourtant avoué, non sans complaisance et sur un mode parfois ostentatoire. » La fureur iconoclaste de Ngoutidé, qui détruit les ayo/yoro, les effigies de Ngakola et le camp d'initiation dans la brousse, le bada, passe par le recours spectaculaire au feu, mais aussi par l'exhibition d'un symbole substitutif, « la croix de Ngoutidé que l'on voit partout », et sa reproduction à travers les petites croix qu'il donnait aux gens qui l'approchaient et qui le considéraient comme un prophète. Cette croix n'est certes pas sans rappeler la canne-croix de Harris, et peut-être aussi le petit bâton courbé, en forme d'une manche de houe, qui était le symbole distribué à ses acolytes par le prophète gbaya Karnu (Burnham \& Christensen 1983 : 4) — l'inspirateur de la révolte anticoloniale Kongo-Wara des années 1920, plus proche géographiquement de Ngoutidé mais avec lequel, nous semble-t-il, le prophète banda partage peu de points communs. C'est un autre «fil» que nous voudrions suivre ici, en considérant la figure de Boganda parmi les influences fortes qui ont contribué à structurer l'itinéraire personnel de Raymond-Ngoutidé. Il n'est d'ailleurs pas impossible d'imaginer une rencontre entre l'abbé, affecté à Bambari, et le jeune Raymond, alors âgé de six ou sept ans : en effet, Boganda organisait des tournées à Bambari et dans les villages des alentours, au cours desquelles il ramassait des fétiches, se faisant accompagner par des enfants de la localité, ses élèves, parmi lesquels des «cousins » de Raymond $^{30}$. Voici ce que Boganda écrivait à Mgr Grandin le 16 juin 1942, de retour d'une de ces tournées à travers ces mêmes villages, dont Lioua, où Raymond résida momentanément pendant sa maladie, puis de nouveau pendant son travail anti-fétichiste et jusqu'à sa mort : "La semaine dernière j'étais sur la route d'Alindao, où les chapelles se sont élevées comme par enchantement. Pas d'images pour y mettre, pas de croix ni de médailles pour remplacer les fétiches que j'enlève aux chrétiens et aux catéchumènes. Ne pourriez-vous pas fouiller le fond de vos armoires pour mes enfants? $»^{31}$. Si l'abbé Boganda réitérait ainsi le «contentieux matériel» sur le lien entre le corps (et le corps du Christ aussi), les choses et le pouvoir qui a gouverné le rapport entre les missionnaires et les populations locales, ici et ailleurs en Afrique équatoriale (Tonda 2005 : 12), nous reviendrons plus loin sur

30. Informations recueillies à Bambari en 2008 auprès de Jean-Pierre Frameau, né en 1930, élève de Boganda, puis compagnon du président pendant son aventure politique, et lié à Ngoutidé par un rapport de «cousinage ».

31. Barthélémy Boganda, Lettre manuscrite à Mgr Grandin, Bambari, le 16 juin 1942, AGCdSE, Fond De Banville, chemise 45.11. L'intégralité de cette lettre est présentée par PÉNEL (1995: 87-88). 
la figure du président centrafricain pour éclairer une dimension proprement politique de son geste iconoclaste.

Le récit de l'entreprise de Ngoutidé, nous l'avons vu, s'enrichit de détails qui plongent le personnage prophétique dans des situations d'épreuve d'où le caractère transcendant de sa vocation doit sortir renforcé. En s'inspirant des récits bibliques et de la persécution du Christ, du destin tragique de Simon Kimbangu, des emprisonnements d'André Matswa et de Simon Pierre Mpadi, le récit de l' «événement Ngoutidé » abonde de références à l'hostilité des représentants du pouvoir, à la fois politique national (des gendarmes que le président Dacko aurait envoyé dans la Ouaka pour capturer le prophète, ou pour lui enlever ses croix), et du pouvoir des Blancs, ici représenté sous son aspect religieux et scientifique: des membres de l'Église catholique auraient envoyé la croix du prophète à Rome, et des médecins se seraient acharnés dès sa première maladie sur le corps de Raymond, éventuellement en essayant de le tuer par des injections d'eau.

Malgré les exagérations évidentes, il est possible que ces détails illustrent les tensions suscitées par cette entreprise anti-fétichiste et, surtout, le succès grandissant attesté par les centaines de personnes qui rejoignaient le village Lioua où Raymond résidait. Mais, au-delà du rapprochement avec le Christ et les persécutions endurées par les prophètes ngunzistes, les éléments inventés a posteriori semblent se multiplier : les Archives spiritaines ne font aucune allusion à ce genre d'événements, mais ils s'interrogent davantage sur le changement de régime intervenu avec la prise de pouvoir de Bokassa le 31 décembre 1965. De même la presse centrafricaine de l'époque ${ }^{32}$, pourtant attentive aux chroniques de l'intérieur du pays, ne fait pas mention de tensions particulières intervenues dans la région de Bambari à cette période. Il n'est donc pas étonnant de retrouver Ngoutidé parmi les protagonistes des «contes centrafricains» recueillis dans la région de la Ouaka par l'écrivain Faustin Ipeko-Etomane dans les années 1970 et publiés en France en 1979. L'itinéraire de Raymond - appelé ici «l'illuminé de Lioua »- est retracé dans trois pages, suivies par des contes ayant comme protagonistes des animaux anthropomorphisés. Sa «persécution » et la fin de la prédication y sont décrites comme il suit : "Dans cette entreprise si délicate de sauveur d'âmes, tout comme le Seigneur Jésus Christ, Aubalé N'Goutédé fut trahi, livré à la justice des hommes comme agitateur », et un peu plus loin, «sa renommée s'effrita. Il s'effaça, optant désormais pour les compétitions vélocipédiques » (Ipeko-Etomane 1979 : 11). L’investissement et les succès de Raymond dans l'activité sportive sont confirmés par la vieille attestation que nous avons retrouvée dans sa maison à Lioua : surtout ils entrent une fois de plus dans un scénario commun à d'autres figures prophétiques de l'époque, dont celle de Simon Zéphirin Lassy, né

32. D'après une première recherche dans les documents conservés aux Archives d'OutreMer. Il est particulièrement difficile de repérer des documents qui remontent aux années immédiatement successives à l'accès du pays à l'indépendance. 
en 1915 près de Pointe-Noire, inspirateur du mouvement de nzambi-bougie, aujourd'hui Église lassyste, qui se distinguait par ailleurs pour ses qualités athlétiques et de boxeur. Bien sûr, cette régénération par l'activité sportive, dans laquelle Raymond obtint certainement des succès, joue dans le récit de l' «événement Ngoutidé » le rôle d'un énième et dernier ingrédient du caractère exceptionnel d'une trajectoire biographique que les Banda continuent de renouveler et d'enrichir, avec attachement, depuis désormais plus de quarante-cinq ans.

NGOUTIDÉ SUR SON VÉLO

Image conservée dans la maison du prophète au village Lioua, sur l'axe Bambari-Alindao.

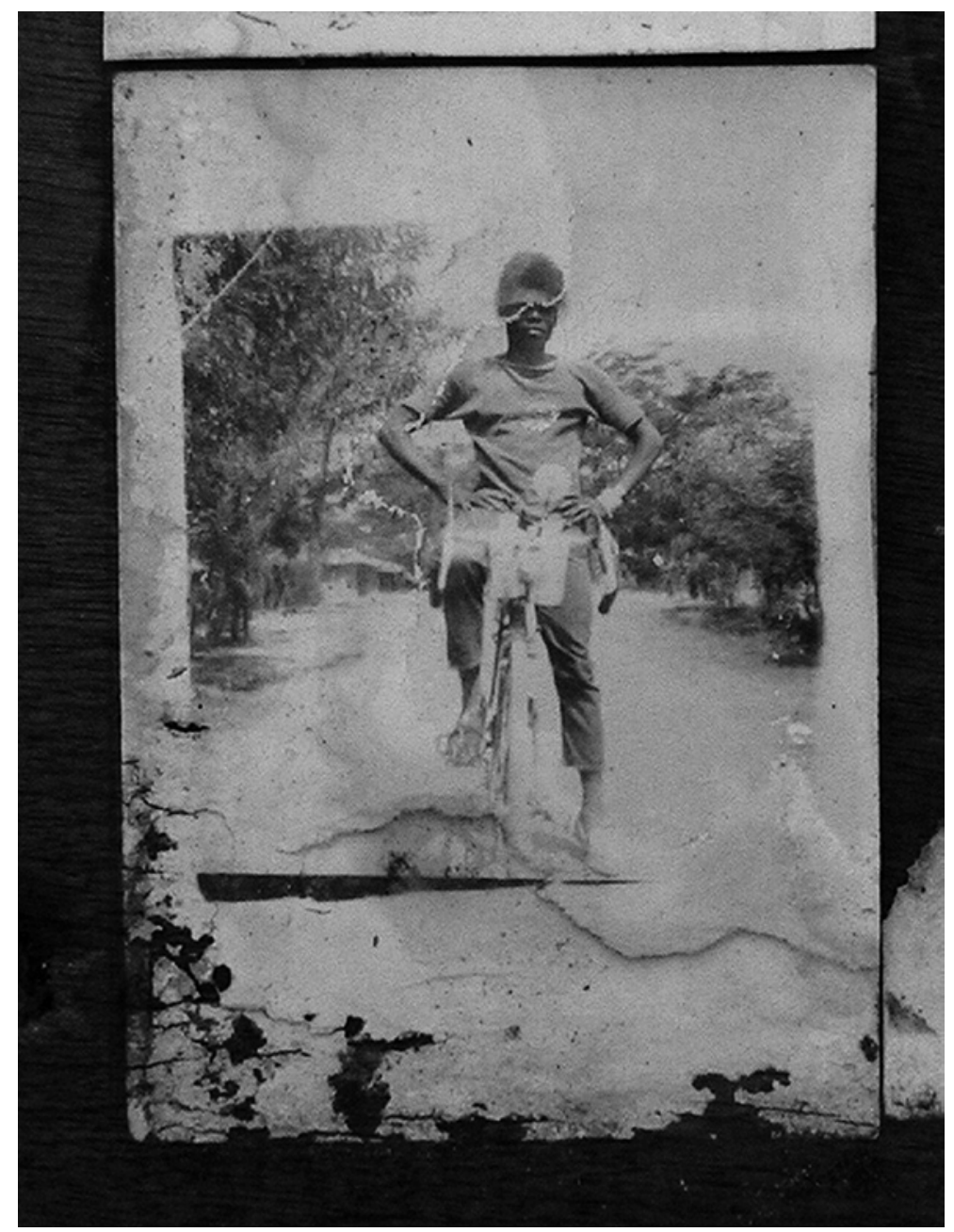




\section{Le récit et ses locuteurs}

L'itinéraire de Raymond Gonemba-Obal répète un scénario devenu classique auquel il apporte néanmoins quelques éléments novateurs, qui résultent de la rencontre entre l'opposition à un culte régional particulier - le semalì - et la réactivation de la figure de Barthélémy Boganda. Cette dernière, attend, par ailleurs, des études approfondies qui mettent en lumière ses nombreuses contradictions ${ }^{33}$. La pérégrination de Ngoutidé, de la périphérie de Bambari aux villages situés le long de la route qui mène à Alindao, puis dans la région comprise entre les localités de Bakala, Grimari et Kouango (où il guérit), semble suivre, avec une étonnante précision, la trace des déplacements de l'abbé Boganda qui, vingt ans plus tôt, résidait à Bambari et était responsable du secteur Bakala-Grimari-Kouango. Dans les années 1960, Raymond, ce formidable athlète banda, pouvait se déplacer à vélo, alors que Boganda l'avait fait sur une mobylette, un geste sportif qui n'est pas sans rappeler l'obstination avec laquelle l'un des premiers missionnaires dans la région, le père Daigre, sillonnait et prêchait dans les villages banda — parcourant à vélo les mêmes pistes - après la destruction par la foudre de la première mission catholique de Bambari, le 20 septembre 1915 (Toso 1994 : 85).

Mais il ne s'agit pas seulement ici de répétition, de reprise, de réinterprétation. L'action de Raymond n'a jamais donné lieu à un «ngoutidisme », et elle est totalement dépourvue d'une certaine dimension collégiale constitutive de l'expérience des ngunza (Mary 2010 : 233). Son efficacité — qui se mesure « en creux » aujourd'hui par la disparition totale d'anciens objets cultuels dans le pays banda (Ceriana Mayneri 2007) — inscrit plutôt cette figure hors-norme parmi ces petits prophètes « en leur pays » (Mary 2000 : 87) qui, à travers la lutte contre les fétiches et la sorcellerie, essaient de «se bricoler une légitimité d'emprunt qui les place en situation de concurrence non seulement par rapport au prêtre-missionnaire, mais également par rapport au chef, devin-guérisseur ou contre sorcier » (ibid. 1993 : 621). Nous analyserons ici l'ampleur et le résultat de la lutte obstinée de Ngoutidé contre les loges semalì, les initiés à Ngakola, les objets de culte (ayo) que les missionnaires et Boganda avaient tenté, en vain, de détruire.

Les données relatives à la vie de Raymond, nous l'avons vu, proviennent de sources peu sûres : un cahier manuscrit conservé au village Lioua, écrit dans le but évident de rapporter la version orale des événements diffusée dans la région depuis des décennies, et un «conte légendaire » de trois pages, à côté d'autres contes dont le style est inspiré des Fables de La Fontaine. Les archives offrent des documents précieux, parfois émouvants, mais n'égalent pas la richesse des matériaux produits dans d'autres

33. Contradictions qui n'avaient pas échappé à Éric DE DAMPIERRE (1967 : 576-577). Outre les travaux de Pierre Kalck et de Jean-Dominique Pénel déjà cités, on se référera utilement aux remarques de BIGO (1988). 
régions de l'Afrique équatoriale. Restent, bien sûr, les récits oraux que le chercheur peut recueillir assez facilement dans le pays banda ou dans certains quartiers de Bangui. Mais le détour par le récit, écrit ou oral, impose désormais d'interroger l'historiographie de la scène prophétique (Johnson 1994 : ix), c'est-à-dire la façon dont les événements des années 1960 sont réinterprétés selon les aspirations et les préoccupations de la population qui en transmet le récit. Il nous faut, en d'autres termes, prêter attention à la manière dont le passé est «mis en intrigue » et les événements sont sélectionnés, agencés de manière rétrospective dans un récit à travers lequel l'«événement» manifeste désormais un surplus de sens (Ricœur 1983).

Au fil de notre reconstitution, Ngoutidé disparaît donc de la scène principale, il devient un comparse muet. Le maître d'œuvre de la mise en intrigue, celui qui sélectionne les événements, organise et explicite ses actes, est la voix collective de la population banda qui, dans les années 1960, choisit de remettre ses ayo à son prophète pour qu'il les brûle. Il n'y eut cependant aucun « disciple » ni, semble-t-il, de successeur à Ngoutidé qui demeure un personnage silencieux auquel les narrateurs du récit ont fait dire, à travers le temps, ce qui correspondait à leurs propres aspirations. Aucun récit autobiographique n'est attesté, et aucune tentative de mise en récit de son expérience ne semble avoir été esquissée par Raymond lui-même au cours de sa vie : au contraire, selon le missionnaire qui officia lors de ses funérailles, il «ne parlait absolument jamais $»^{34}$ de sa vie des années 1960. Et selon son frère cadet et sa dernière femme, il n'aurait jamais donné d'explications sur son retour à la vie de cultivateur, à Lioua. Dans le Journal de Bakala du 31 décembre 1964, le missionnaire de la localité écrivait que Ngoutidé «n'a appelé personne [...] il a suffi qu'il ouvre la bouche pour qu'on lui obéisse », et les routes étaient effectivement pleines de Banda «allant spontanément vers lui ». Mais cette voix du prophète et son contenu nous ne l'entendrons jamais. Ngoutidé apparaît alors moins comme un personnage charismatique, au sens weberien, que l'instrument d'une herméneutique locale qui réactive, à chaque répétition du récit, le souvenir de l'abandon du «fétichisme » des ancêtres et de l'adhésion collective au christianisme. C'est donc à partir des aspirations de ce locuteur collectif que nous interrogerons à nouveau le sens des événements des années 1960.

\section{Des discours de la dépossession}

En Centrafrique, aujourd'hui, la prégnance de la sorcellerie comme grille explicative du malheur se greffe sur une réalité sociodémographique particulièrement complexe et s'exprime par une crise étiologique qui investit tous les secteurs sociaux, politiques et professionnels du pays. De la presse banguissoise aux rumeurs qui circulent en milieu rural, des églises (Fancello

34. Père Louis Sénéchal, communication personnelle du 25 juin 2007. 
2012) aux tribunaux (Cimprič 2012), des «cliniques» des tradipraticiens aux hôpitaux et facultés universitaires (Ceriana Mayneri 2012), les soupçons et les accusations de sorcellerie se multiplient et envahissent les espaces publics et privés de la vie centrafricaine. De ce point de vue, le pays suit un parcours de reformulation et de réinvention incessantes des représentations de la sorcellerie, dont les dimensions et certains caractères novateurs sont attestés par différents contextes africains (Geschiere 1995; Comaroff \& Comaroff 1999 ; Ashforth 2005 ; Fancello 2008 ; Bonhomme 2011). Tout en s'inscrivant dans le schéma classique qui fait du village le foyer de la sorcellerie, et en insistant sur une recrudescence de la menace sorcellaire qui est un thème récurrent depuis l'époque coloniale (Mary 2010 : 139), la fréquence et la dureté des peines prononcées par les tribunaux centrafricains à l'encontre de présumés sorciers, ainsi que le recours à la violence populaire, témoignent de l'exacerbation des tensions qui accompagne les phénomènes de sorcellerie dans ce pays équatorial. Mais, la condamnation sans appel des fétiches et de la sorcellerie, prononcée en Centrafrique aussi par les Églises pentecôtistes et les groupes charismatiques (Fancello 2012), ne peut occulter la polysémie de ces yoro - en banda, ayo — dont il semble urgent d'approfondir ici l'ambivalence. Le père Tisserant a certainement su éclairer pour la première fois, en 1931, la polysémie du terme ayo, mais il revient à Eggen (1976: 48c,d) le mérite d'avoir contextualisé la trajectoire de ce concept par rapport à la disparition des anciens cultes banda, à une époque où ces derniers étaient abandonnés sous l'impulsion, entre autres, de Ngoutidé.

Selon un schéma répandu dans la région équatoriale - et étudié en profondeur par E. E. Evans-Pritchard à la suite de son séjour parmi les Azandé - en cas de malheur, de maladie ou de mort imprévue, les Banda s'orientent, à travers le recours aux oracles, vers la recherche d'un responsable, un «sorcier» qui, en raison de sa malveillance et en déclenchant des pouvoirs extrahumains ou en préparant des poisons, aurait provoqué ces événements. Il s'agit, en d'autres termes, d'une étiologie mystique, dont il convient de rappeler ici, à la suite d'Evans-Pritchard (1972: 38) qu'elle ne postule pas tant l'existence de pouvoirs mystérieux et exceptionnels, mais qu'elle s'efforce plutôt de circonscrire le champ du «bon sens » et d'interpréter des événements communs à partir de leur observation, éventuellement en complétant l'explication fournie par l'étiologie naturelle (ibid. : 103104). Ici, en tout cas, notre attention s'oriente moins sur les caractéristiques de ce «sorcier", en banda eyiondro - qui semble par ailleurs cumuler les caractères qu'Evans-Pritchard attribuait soit au pouvoir psychique et involontaire du witch, soit aux techniques de manipulation des «médecines » du sorcerer - que sur la lutte et les systèmes de protection contre le danger sorcellaire omniprésent. L'ayo, en tant que remède d'origine végétale, voire, aujourd'hui, médicament pharmaceutique, fait son apparition lors d'un diagnostic. Immédiatement ses significations s'étendent, se multiplient, pour désigner non plus seulement un objet thérapeutique mais aussi une pratique. 
Le père Tisserant (1931: 364) était clair à ce propos, lorsque parmi les traductions de ayo (il écrivait plutôt oyo) il proposait "pratique fétichiste servant de remède ». Néanmoins, Eggen (1976 : 48b) est beaucoup plus précis lorsqu'il suggère l'existence d'un lien entre une plante, le pouvoir curatif qu'on lui attribue, et l'«esprit » associé à ce pouvoir curatif : c'est donc un eyiayo - eyi, "celui », qui possède l'ayo - qui maîtrise ce pouvoir curatif via le rapport personnel qu'il entretient avec l'esprit, et qui l'administre, ou qui en fait un usage antisocial en détournant « le pouvoir à son profit et contre les autres » (ibid.). Pour faciliter la lecture et l'analyse nous choisissons de distinguer par la suite deux modalités d'acquisition de ce pouvoir/ayo, et deux manières de l'exercer, sachant que, probablement, dans l'ancienne société banda ces fonctions se recoupaient.

Les ayo jouaient un rôle majeur dans le culte de Ngakola et lors des initiations aux loges semalì. Pendant plusieurs mois, les néophytes - des garçons et des jeunes filles, regroupés ensemble - séjournaient dans un camp apprêté à l'écart du village, le bada, et recevaient des enseignements sur la vie d'adulte, sur l'organisation de la société, et sur le culte voué à Ngakola, l'esprit de la brousse. Ces néophytes étaient oints avec des médicaments, des ayo, pour les protéger contre la sorcellerie, mais ils recevaient aussi des enseignements sur l'utilisation des ayo, de telle manière que, dans la première moitié $\mathrm{du} \mathrm{Xx}^{\mathrm{e}}$ siècle, il n'était pas rare qu'un initié semalì possède son propre camp bada et que là il consulte Ngakola au sujet d'une maladie ou d'une infortune, en passant par l'intermédiaire de l'oracle. Nous résumons ainsi une trajectoire bien plus complexe, s'étalant sur plusieurs décennies, qui a profondément changé l'organisation et les fonctions du culte de Ngakola (Grootaers 2007 : 23-24, 66-67). Mais ce que nous tenons à souligner ici est le fait que, malgré certaines malversations et la violence exercée contre les non-initiés, le culte était encore, au lendemain de l'indépendance, un recours contre la maladie : on s'adressait à Ngakola ou à sa «femme » Yamissi, pour connaître l'origine d'une affliction ou obtenir les médicaments appropriés, et on initiait les enfants malades à Ngakola afin d'en obtenir la guérison ${ }^{35}$. En revenant à la fin des années 1970 sur le concept banda de ayo et sur le culte de Ngakola, Eggen (1979: 179) a même écrit que le culte semalì dans son ensemble peut être considéré comme un ayo, dans la mesure où l'intervention de l'esprit de la brousse et le recours aux remèdes manipulés par les initiés étaient deux stratégies recherchées pour obtenir une guérison ou pour se protéger contre des malheurs futurs.

Nos observations nous conduisent à faire état d'une deuxième modalité d'acquisition de l'ayo, et d'une manière différente de profiter de son autorité et exercer son pouvoir. Parmi les Banda, la causalité qui, aujourd'hui est

35. Ces informations recueillies sur le terrain nous ont été confirmées par le linguiste Marcel Diki-Kidiri, originaire de Bambari (communication personnelle du 20 septembre 2009). 
attribuée à la sorcellerie, procède d'un système de représentations et d'interprétation de la réalité plus articulé, qui manifeste par ailleurs certaines différences par rapport au cas zandé illustré par Evans-Pritchard. Si, d'un côté, il est vrai que derrière la maladie, et les malheurs de la vie en général, on soupçonne l'action d'une force extrahumaine, d'un autre côté ces malheurs ne correspondent pas nécessairement à une attaque en sorcellerie, mais peuvent être aussi le signe de l'intervention d'une force invisible qui agit à la suite de l'infraction d'une règle sociale ou familiale, ou pour établir un rapport avec la personne affectée. Eggen (1976:48b), qui nous offre la première et unique tentative de systématisation de ces données diverses, écrit significativement : «De fil en aiguille, on finit pas considérer la maladie comme le moyen par lequel le génie se révèle pour choisir son intermédiaire. »Et, d'après cet auteur, lorsque un oracle proclamait qu'une maladie était occasionnée par la présence d'une telle puissance, trois stratégies diverses se présentaient au malade : soit il procédait à des offrandes pour écarter l'esprit, soit il quittait son groupe familial et se faisait «adopter » dans une autre famille - selon une coutume sur laquelle nous ne pouvons pas nous attarder ici - soit, finalement, il « intériorisait l'esprit en acceptant son élection comme adepte ou comme dignitaire» (ibid. : 48/e). Nous voilà de nouveau devant cet eyi-ayo, celui qui détient un pouvoir ou une connaissance particuliers, le «dignitaire d'une puissance extrahumaine » (ibid.) se manifestant, éventuellement, par la maladie.

Le lexique des missionnaires et leurs gestes ont certainement agi sur le choix que fit Raymond Gonemba-Obal de détruire les «fétiches » banda. Aujourd'hui, ce lexique, exacerbé en Centrafrique par l'influence grandissante de l'ethos pentecôtiste, réapparaît dans les discours de nos interlocuteurs lorsqu'ils évoquent la lutte que «le plus grand des Banda » mena contre le «fétichisme» des ancêtres. Mais cette insistance sur le fétiche et le fétichisme, sans nous enfermer dans la seule dimension religieuse de l'entreprise de Ngoutidé, recèle des indications précieuses sur la signification politique et les enjeux réels de cette trajectoire iconoclaste. La figure de Barthélémy Boganda, qui à Bambari s'élevait contre le fétichisme banda, révèle des contours plus précis, s'inscrivant déjà à l'époque de son ministère dans une dialectique du religieux et du politique qui visait non seulement la dissolution des «superstitions » indigènes, mais surtout la désarticulation de l'organisation sociale et politique des sociétés équatoriales. Ce n'est pas un hasard si Félix Éboué, cette figure de premier plan de la colonisation française, envoyé comme administrateur parmi les Banda en 1909, perçut l'enjeu qu'il y avait à s'accorder avec les loges semalì pour pouvoir exercer un premier contrôle sur un territoire qui était alors loin d'être pacifié (Weinstein 1978), et revint successivement dans certains de ses écrits sur le culte de Ngakola et les fonctions de ses adeptes (Éboué 1931). Lorsque Raymond Gonemba-Obal guérit et décide de brûler les fétiches du génie 
de la brousse, il répète sans nul doute le geste iconoclaste des missionnaires, et peut-être le geste de Boganda, auquel il aurait pu assister au début des années 1940, mais il reste enfermé dans les contradictions politiques du rôle qu'il assumait alors et pour les quelques années à venir. Ce jeune homme banda s'attaquait à un dispositif cultuel qui avait contribué à maintenir la cohésion des groupes banda au moment du passage du régime esclavagiste au régime concessionnaire, et qui demeurait après l'indépendance un recours contre les malheurs, une protection contre la maladie et, aussi, contre la malveillance des sorciers. En d'autres termes, Ngoutidé entreprit avec véhémence de démanteler le système basé sur l'autorité des ayiayo, il contesta leur influence, il brisa les symboles matériels de leur pouvoir. Et c'est ici, dans cette contestation, que la figure du prophète banda manifeste les signes d'une contradiction irrésolue, qui décrète aussi, en quelque sorte, l'échec de la mission dont il se croyait investi. «Une relation de complicité dans l'adversité » : c'est dans ces termes qu'André Mary (1993 : 624) décrit le cercle des rapports du prophète et du sorcier, autrement dit le fait que non seulement le deuxième conforte le premier dans sa mission - ce qui constitue aussi le dilemme du nganga, du devin-guérisseur, du contre-sorcier qui est toujours considéré très, trop proche du pouvoir sorcier qu'il dit combattre - mais surtout le fait que le prophète «donne à lire son pouvoir dans les termes de ce langage de la force que d'une certaine façon il récuse » (ibid. : 626). En se réveillant après une longue maladie, et en affirmant que c'était le baptême qui l'avait guéri ${ }^{36}$, Ngoutidé a accepté cette «élection » selon un schéma qui, nous l'avons vu, était loin d'être nouveau même parmi les Banda, en devenant de surcroît l'un de ces ayiayo contre lesquels il combattait. Double échec, d'une certaine manière, puisque, outre l'impossibilité de sortir de la logique qui oblige celui qui combat le pouvoir des ayo à maîtriser un pouvoir dont la nature et l'intensité ne sont pas vraiment différentes, le résultat de cette destruction par le feu a été de priver la population d'un remède ancestral - à la lettre, des ancêtres — contre le malheur et ses causes sorcellaires.

Précisons néanmoins un dernier aspect. L'échec auquel nous nous référons est moins celui, entièrement personnel, d'un homme qui combat ce même pouvoir qu'il possède (ou dont il est possédé), que celui très concret d'une communauté d'individus qui a fait confiance à son prophète et qui n'a plus cessé de souffrir des conséquences de ce choix, notamment à travers la perception d'une recrudescence de la sorcellerie. C'est ici le sens du glissement, d'une dimension biographique à la dimension collective, que nous avons proposé de suivre en appréhendant l'événement Ngoutidé à partir des préoccupations du locuteur de son récit. En effet, pour Raymond Gonemba-Obal, nous l'avons vu, un certain réinvestissement après la fin

36. Journal de Bakala du 31 décembre 1964. 
de la mission n'a pas été impossible : les succès sportifs, le rapport avec des commerçants étrangers, la grande maison «en dur » de Lioua, témoignent d'une capacité à se réinventer une vie nouvelle - bien que le sens de sa dernière maladie demeure, de ce point de vue, certainement troublant et entièrement à élucider. Mais, la trajectoire de la population banda a été différente et non moins problématique. La destruction massive des anciens remèdes s'est accompagnée, en pays banda et ailleurs en Afrique centrale, de leur condamnation sans appel : à travers la prédication missionnaire et l'opposition de l'Administration d'abord, et par le recours au feu purificateur de Ngoutidé ensuite, les ayo des anciennes sociétés initiatiques, ceux des ancêtres, ont été reconnus comme des objets sorcellaires et démoniaques. Leur réversibilité - le fait qu'ils pouvaient servir à des fins socialement constructives tout comme à des fins malveillantes - a été interrompue : les ayo de Ngakola ont progressivement perdu leur efficacité thérapeutique et leur versant antisocial est devenu prépondérant. Il en résulte une exacerbation dans la perception de la menace sorcellaire et le sentiment d'être dépourvus des moyens de s'opposer efficacement aux dangers, réels et imaginaires, d'un monde caractérisé par une insécurité grandissante. Cette perception d'une « dépossession » de connaissances et de pouvoirs ne coïncide que partiellement avec la «déconnexion» du monde moderne que d'autres auteurs ont perçue sur leurs terrains africains (Ferguson 2008 : 1112; Comaroff \& Comaroff 1993: xii), et qui est certainement l'un des thèmes forts qui ont traversé différentes études récentes consacrées aux représentations de la sorcellerie en Afrique (Geschiere 1995; De Boeck 2008). Puisqu'on ne peut être dépossédé que de quelque chose dont on disposait au préalable, ou dont on croyait disposer au préalable, la dépossession renvoie en même temps au sentiment d'une absence vécue dans le présent, et à un passé réinterprété ou réinventé à partir des déceptions et aspirations du temps présent. Par ce terme de « dépossession » nous voulons signifier la nécessité d'appréhender l'actualité des représentations de la sorcellerie à partir de ce qu'elles nous disent sur la manipulation de la mémoire et la réinvention du passé récent. C'est à Bambari, auprès d'un technicien supérieur de la santé, qui fut aussi un témoin des dernières initiations semalì, que nous avons recueilli cette lamentation sur la disparition des pouvoirs et connaissances des ancêtres :

« Chez vous, les Blancs, vous avez des savants qui font des découvertes et qui les mettent à la disposition de l'humanité [...]. Or, il se trouve qu'en milieu banda nous avons certaines potentialités, nous avons certains pouvoirs. Ce sont des choses qui ont existé en milieu banda et qui malheureusement sont en train de disparaître [...]. Pour te montrer ça, il faudrait que tu passes par les choses de semalì, de Ngakola, et c'est en ce moment qu'on peut te montrer certains secrets. Ce sont des choses qui ont existé, en principe si nos grands-parents n'étaient pas [...] un peu égoïstes, qu'ils mettaient ça à la disposition de la communauté banda [...]. Malheureusement ces choses-là, qui ont des potentialités exploitables, sont restées [...] sous un grand secret, et maintenant c'est disparu. » 
L'anthropologue Tamara Giles-Vernick (1996 : 246) ${ }^{37}$, qui a analysé la manière dont les événements passés sont «mis en intrigue » dans les récits que les Banda m'bres, habitants la préfecture de Nana-Gribizi, au nordouest de la Ouaka, proposent des anciens rituels d'initiations, écrit : «Banda represented themselves as metamorphosing from a loosely organized people living in remote, scattered settlements, to a colonized people living along "big roads", to a Christian people, and most recently, to a dispossessed people. »Quelques deux cents kilomètres plus à l'Est, les groupes banda de la Ouaka dénoncent avec amertume la disparition des connaissances et pouvoirs, les ayo, des anciens initiés semalì, sans pour autant relativiser la glorification rétrospective $\mathrm{du}$ «prophète banda international ». La contradiction de ce souvenir manifeste selon nous les doutes et inquiétudes qui continuent d'accompagner la mémoire collective de cette population centrafricaine qui choisit, tout au long du $\mathrm{Xx}^{\mathrm{e}}$ siècle, d'abandonner progressivement ses cultes et d'adhérer à la religion des missionnaires. Depuis plus de quarante ans, des hommes et des femmes de la Ouaka renouvellent le récit de la vie du prophète banda. À chaque répétition, et selon les aspirations des narrateurs, de nouveaux détails s'ajoutent, mais aussi des contradictions et des non-dits qui constituent une voie d'accès privilégiée à la compréhension des tensions et des aspirations qui travaillent la population de cette région centrafricaine.

Institut des mondes africains (IMAF), Paris.

\section{BIBLIOGRAPHIE}

AsCH, S.

1983 L'église du prophète Kimbangu. De ses origines à son rôle actuel au Zaïre, Paris, Karthala.

AshForth, A.

2005 Witchcraft, Violence, and Democracy in South Africa, Chicago, Chicago University Press.

BALANDiER, G.

1955 Sociologie actuelle de l'Afrique Noire, Paris, PUF.

BIGO, D.

1988 Pouvoir et obéissance en Centrafrique, Paris, Karthala.

37. Parmi les Banda m'bres, Tamara Giles-Vernick n'a jamais entendu parler du prophète Ngoutidé (communications personnelles du 13 avril et 5 mai 2008). 
BonHOMme, J.

2011 «Les numéros de téléphone portable qui tuent. Épidémiologie culturelle d'une rumeur transnationale », Tracés, « Contagion/Contamination », 21 : 125-150.

BouQuiaux, L.

1978 Dictionnaire sango-français, Paris, SELAF.

Burnham, P. \& Christensen, T.

1983 «Karnu's Message and the "War of the Hoe Handle": Interpreting a Central African Resistance Movement », Africa : Journal of the International African Institute, 53 (4) : 3-22.

Ceriana Mayneri, A.

2007 «La statuaire Banda. État des lieux et patrimoine », in B. MARTinELli (dir.), Patrimoine esthétique et artistique centrafricain, Bangui, UNESCO : 135156.

2012 «Soigner, guérir, convertir. Les étudiants en médecine de Bangui (République centrafricaine) et leur rapport à la médecine traditionnelle : une analyse du discours », Psychopathologie africaine, XXXV (3) : 277-308.

CIMPRIČ, A.

2012 «Le magistrat et le sorcier. Les talimbi devant le tribunal centrafricain », in B. Martinelli \& J. Bouju (dir.), Sorcellerie et violence en Afrique, Paris, Karthala : 131-152.

Comaroff, J. \& Comaroff, J. L.

1993 «Introduction », in J. Comaroff \& J. L. Comaroff (eds.), Modernity and its Malcontents : Ritual and Power in Postcolonial Africa, Chicago, The University of Chicago Press.

1999 «Occult Economies and the Violence of Abstraction : Notes from the South African Postcolony », American Ethnologist, 26 (2) : 279-303.

COQUERY-VIDROVITCH, C.

1972 Le Congo au temps des grandes compagnies concessionnaires, 1898-1930, La Haye-Paris, Mouton \& Co.

CORDELl, D.

1985 Dar al-Kuti and the Last Years of the Trans-Saharan Slave Trade, Madison, The University of Wisconsin Press.

1999 «Des "réfugiés" dans l'Afrique précoloniale ? L'exemple de la Centrafrique, 1850-1910», Politique Africaine, 85 : 16-28.

De Boeck, F.

2008 « Kinshasa. Tales of the Invisible City \& the Second World », in P. GEschiÈRE, B. Meyer \& P. Pels (eds.), Readings in Modernity in Africa, London, The International African Institute : 124-135.

De DAMPIERre, É.

1967 Un ancien royaume Bandia du Haut-Oubangui, Paris, Plon. 
DesRoche, H.

1963 «Les messianismes et la catégorie de l'échec », Cahiers internationaux de sociologie, XXXV : 61-84.

Dozon, J.-P.

1995 La cause des prophètes. Politique et religion en Afrique contemporaine, Paris, Édition du Seuil.

ÉBOUÉ, F.

1931 «Les sociétés d'initiation en pays Banda (à l'occasion d'un jugement récent) », Bulletin de la Société de recherches congolaises, 13: 3-16.

EGGEN, W.

1976 Peuple d'autrui, Bruxelles, Pro Mundi Vita.

1979 «Le faux refuge. Diable, sorcellerie et résidence chez les Banda », Cultures et Développement, 11 (2) : 175-192.

Evans-Pritchard, E. E.

1972 Sorcellerie, oracles et magie chez les Azandé, Paris, Gallimard.

FANCELLO, S.

2006 Les aventuriers du pentecôtisme ghanéen. Nation, conversion et délivrance en Afrique de l'Ouest, Paris, IRD-Karthala.

2008 «Sorcellerie et délivrance dans les pentecôtismes africains », Cahiers d'Études africaines, XLVIII (1-2), 189-190 : 161-183.

2012 « D'un guérisseur à l'autre : diagnostic, délivrance et exorcisme à Bangui », in B. Martinelli \& J. Bouju (dir.), op. cit. : 55-83.

FERGuson, J.

2008 "Global Disconnect. Abjection \& the Aftermath of Modernism », in P. Geschière, B. Meyer \& P. Pels (eds.), op. cit. : 8-16.

Geschiere, P.

1995 Sorcellerie et politique en Afrique. La viande des autres, Paris, Karthala.

Giles-Vernick, T.

1996 «Na lege ti guiriri (On the Road of History) : Mapping Out the Past and Present in M'Bres Region, Central African Republic », Ethnohistory, 43 (2) : 245-275.

Grootaers, J.-L.

2007 «Un creuset aux frontières ouvertes en Afrique Centrale», in J.-L. GRoOTAERS (dir.), Ubangi. Art et cultures au cœur de l'Afrique, Arles, Actes Sud.

IPEKo-Etomane, F.-A.

1979 Veillées villageoises. Contes centrafricains, Paris, L'arbre du voyageur.

JEAN, S.

1963 Les Langbas, population d'Oubangui-Chari, Paris, Bureau pour le développement de la production agricole. 
JOHNSON, D. H.

1994 Nuer Prophets. A History of Prophecy from the Upper Nile in the Nineteenth and Twentieth Centuries, Oxford, Clarendon Press.

KALCK, P.

1992 Histoire centrafricaine. Des origines à 1966, Paris, L'Harmattan.

1995 Barthélémy Boganda. "Élu de Dieu et des Centrafricains », Saint-Maur-desFossés, Sépia.

KinatA, C.

2008 «Barthélémy Boganda et l'Église catholique en Oubangui-Chari », Cahiers d'Études africaines, XLIII (3), 191 : 549-565.

LEIRIS, M.

1934 L'Afrique fantôme, Paris, Gallimard.

MACGAFFeY, W.

1983 Modern Kongo Prophets. Religion in a Plural Society, Bloomington, Indiana University Press.

MARCHAL, R.

2009 «Aux marges du monde, en Afrique centrale...», Les Études du CERI, 153-154.

MARY, A.

1993 «Le travail symbolique des prophètes d'Eboga: logiques syncrétiques et entre-deux culturel », Cahiers d'Études africaines, XXXIII (4), 132: 613643.

2000 « Voyage visionnaire et errance prophétique : du nomadisme à la fondation », Social Compass, 47 (1) : 77-92.

2010 Visionnaires et prophètes de l'Afrique contemporaine. Tradition initiatique, culture de la transe et charisme de délivrance, Paris, Karthala.

PÉNEL, J.-D.

1995 Barthélémy Boganda. Écrits et discours. 1946-1952 : la lutte décisive, Paris, L'Harmattan.

PIETZ, W.

2005 Le fétiche. Généalogie d'un problème, Paris, Kargo \& L’Éclat.

Riceur, P.

1983 Temps et récit, tome I, Paris, Éditions du Seuil.

SANTANDREA, S.

1964 A Tribal History of the Western Bahr el Ghazal, Bologna, Nigrizia.

SHANK, D. A.

1994 Prophet Harris, The «Black Elijah » of West Africa, Leiden, Brill.

1999 «Le Pentecôtisme du prophète William Wadé Harris », Archives de sciences sociales des religions, 105 (1) : 51-70. 
Tisserant, R. P. C.

1931 Dictionnaire Banda-Français, Paris, Institut d'Ethnologie.

TONDA, J.

2005 Le Souverain moderne. Le corps du pouvoir en Afrique centrale (Congo, Gabon), Paris, Karthala.

Toso, P. C.

1994 Centrafrique, un siècle d'évangélisation, Bangui, Conférence Épiscopale Centrafricaine.

VELLUT, J.-L.

2005 Simon Kimbangu. 1921 : de la prédication à la déportation. Les sources. Vol. I : Fonds missionnaires protestants (1). Alliance missionnaire suédoise (Svenska Missionsförbundet, SMF), Bruxelles, Académie royale des Sciences d'Outre-Mer, («Fontes Historiæ Africanæ»).

2010 Simon Kimbangu. 1921 : de la prédication à la déportation. Les sources. Vol. I: Fonds missionnaires protestants (2). Missions baptistes et autres traditions évangéliques, Bruxelles, Académie royale des Sciences d'OutreMer («Fontes Historiæ Africanæ»).

WEINSTEIN, B.

1978 «Governor-General Félix Eboué (1884-1944)», in L. H. GanN \& P. DuIGNAN (eds.), African Proconsuls. Europeans Governors in Africa, New YorkLondon-Stanford, The Free Press, Collier Macmillan Publishers \& Hoover Institution.

RÉSUMÉ

Dans les années 1960, parmi les Banda de Centrafrique se produisit une exceptionnelle effervescence anti-fétichiste. Un homme appelé Ngoutidé (" eau froide ») guérit d'une longue maladie et s'adonna à une vaste œuvre de destruction des objets cultuels et des camps d'initiation des anciennes associations fermées banda. Il prêcha la conversion au catholicisme. Depuis plus de quarante ans, la population banda répète le récit de la vie et de la prédication de Ngoutidé, en glorifiant rétrospectivement sa mission d'évangélisation et de destruction des fétiches. Après avoir reconstruit la trajectoire biographique de Ngoutidé - à partir de recherches d'archive et d'entretiens enregistrés en Centrafrique -, nous nous arrêterons sur les ambivalences et les contradictions de cette figure prophétique, ainsi qu'elles émergent dans les récits sur sa vie diffusés en Centrafrique.

\section{ABSTRACT}

Ngoutidé "the Enlightened One of Lioua". Destruction of Fetishes and Representations of Witchcraft in the Central African Republic. - In the 1960s there was an important anti-fetishist movement among the Banda of the Central African Republic. 
A man called Ngoutidé ("fresh water"), after recovering from a long illness, dedicated himself to destroying objects of worship and the places of the ancient initiation societies of the Banda. Ngoutide preached in the name of the catholic faith. For more than forty years now, the Banda population has been continuing to repeat the story of the life and preaching of Ngoutidé, retrospectively praising his mission to evangelize and destroy fetishes. After reconstructing Ngoutidé's biography, starting from research in the archives and interviews carried out in the Central African Republic, this essay dwells on the ambivalences and contradictions of this prophetic figure, as he is described now in the Central African Republic.

Mots-clés/Keywords : Centrafrique, Banda, Ngoutidé, évangélisation, mouvements prophétiques, sorcellerie/Central African Republic, Banda, Ngoutidé, evangelization, prophetic movements, witchcraft. 